\title{
Bacteroides fragilis controls intestinal inflammation through promoting Treg differentiation via TGF- $\beta /$ Smad3 signaling pathway
}

\author{
Menghui Fan \\ China Pharmaceutical University

\section{Zhenxing Zhang} \\ China Pharmaceutical University

\section{Guojing Ruan} \\ China Pharmaceutical University
}

\section{Fang Wei}

China Pharmaceutical University

\section{Xinyue Gu}

China Pharmaceutical University

\section{Tao Xi}

China Pharmaceutical University

\section{Yingying Xing ( $\square$ cpuskyxyy@126.com )}

China Pharmaceutical University https://orcid.org/0000-0001-5566-3096

\section{Research Article}

Keywords: Bacteroides fragilis, Inflammatory bowel disease, Microbiota, Regulatory T cells, Smad3

Posted Date: May 13th, 2021

DOI: https://doi.org/10.21203/rs.3.rs-444129/v1

License: (1) This work is licensed under a Creative Commons Attribution 4.0 International License. Read Full License 


\section{Abstract}

Alteration of intestinal flora is usually associated with various gastrointestinal diseases.Microbial homeostasis can enhance host immune tolerance and regulate intestinal immune balance.Our previous studies have found that the peptidoglycan framework of Lactococcus lactis(L.lactis),gram positive enhancer matrix particles (GEM), improved the intestinal flora diversity and significantly increased the relative abundance of Bacteroidetes in colitic mice. Of note,Bacteroides fragilis(B.fragilis) can alleviate inflammation by promoting the expressions of intestinal regulatory $T$ cells (Tregs) and anti-inflammatory cytokines. However, mechanism of $B$.fragilis regulating intestinal immunity remains unclear,thus we here seek to explore the effects of B.fragilis and its mechanism by DSS-induced experimental colitis.Results show that $B$.fragilis contributes to the restoration of gut microbiota composition and an increase in shortchain fatty acids(SCFAs) production of mice with colitis. The treatment mediates the differentiation of naive $\mathrm{CD} 4^{+} \mathrm{T}$ cells into Tregs probably through TGF- $\beta$ activated by SCFAs, and TGF- $\beta /$ Smad 3 signaling is indispensable for both Treg expansion and IL-10 expression. The study demonstrates the immune pathways by which $B$. fragilis controls the inflammatory process and it may be an effective therapeutic option for intestinal diseases.

\section{Introduction}

Intestinal flora is closely related to intestinal mucosal immunity, and the dysregulation of intestinal flora often leads to a variety of intestinal diseases,such as inflammatory bowel diseases(IBD),Crohn's disease(CD) and Ulcerative colitis(UC)[1].Our previous work demonstrated that GEM administration in colitic mice,the peptidoglycan framework of Lactococcus lactis(L.lactis)[2], can restore the gut microbiota diversity, and the most significant is increasing the relative abundance of Bacteroidetes [3]. B.fragilis,one of the most common members of Bacteroidetes, is commonly found in the human gut bacteria, also found in the oral cavity and the upper respiratory tract.The enterotoxin-free strains are considered to be beneficial bacteria and an important part in intestinal flora,which is conducive to maintain gut homeostasis [4].Studies have found that $B$.fragilis can induce the expansion of intestinal Tregs during experimental colitis,thus further to enhance the regulatory immune response in intestinal tract, and then reduce the inflammation [5]. Additionally, B.fragilis colonization can prevent inflammation and tumor formation in a germ-free colitisassociated colorectal cancer(CAC) mouse model via Toll-like receptor 4(TLR4) signal pathway [6].However, the biological mechanisms that $B$.fragilis controls intestinal inflammation are still obscure.Here,we desire to explore the immunomodulatory effects of $B$.fragilis treatment and immune pathways it regulates in colitis.

Treg is a subset of $\mathrm{T}$ cells with immunosuppressive function. Initial $\mathrm{CD} 4^{+} \mathrm{T}$ cells can be induced to differentiate into different $T$ cell subsets under different circumstances, including effector cells Th1, Th2, Th17 and Tregs.Recent studies have shown that Tregs, which produce the anti-inflammatory cytokine IL-10, play an important role in improving immune tolerance [7].However, how B.fragilis regulating the expression of Treg cells during intestinal inflammation remains to be studied. During the differentiation of Tregs, TGF$\beta / \mathrm{Smad} 3$ is a key signal transduction pathway that determines the expression of transcription factor Foxp3, an unique marker on Tregs.Smad3 is the first signal molecule that identifies the signal transmission of TGF- 
$\beta$ and acts after phosphorylation [8,9].Therefore, the regulatory mechanism of TGF- $\beta /$ Smad3 pathway in B.fragilis alleviating colitis was further explored.

In this study, we tested the effects of $B$.fragilis on gut microbiota and intestinal immune with DSS-induced colitis. Results indicated that oral administration of $B$.fragilis conserved the gut microbiota diversity and enhanced SCFAs production. More importantly,the expansion of Tregs in B.fragilis-treated mice was dependent on TGF- $\beta /$ Smad3 signaling pathway and provided protection from followed colitis. While in mice with smad3 knocked down, the results highlighted that smad3 was indispensable for immune modulation induced by B.fragilis. These data demonstrated the capability of B.fragilis to effectively control intestinal inflammation and be considered as a valuable option in intestinal-related pathologies.

\section{Materials And Methods}

\section{Mice}

$20 \pm 2 \mathrm{~g}$, female, specific-pathogen-free (SPF) C57BL/6 mice,purchased from Nanjing Qinglongshan Animal Breeding Center and the Comparative Medicine Center of Yangzhou University,were housed in the Animal Experimental Center of China Pharmaceutical University.Mice were randomly divided into different experimental groups after one week adapting.

\section{Bacteria culture}

B.fragilis ATCC25285(Beina Biotechnology) was cultured on colombian medium with $10 \%$ degenerated sheep blood(Jiulong Biotechnology) under anaerobic condition at $37^{\circ} \mathrm{C}$ for $2-3$ days. Helicobacter pylori NSH57(Nanjing Medical University) was cultured on Helicobacter pylori solid medium(Qingdao Hope Biotechnology) with 10\% Fetal Bovine Serum(FBS,Gibco) and 1\% H.pylori bacteriostat(Qingdao Hope Biotechnology) under microaerophilic condition at $37^{\circ} \mathrm{C}$ for $3-4$ days.

\section{H.pylori colonization}

Mice were deprived of water and fasted for eight hours and then treated with $1 \times 10^{9} \mathrm{CFU}$ H.pylori by oral gavage in interval of two days for three times to induce gastritis. Next,mice were inoculated with $1 \times 10^{9} \mathrm{CFU}$ B. fragilis for 3 days. When sacrificed,20 mg fresh gastric tissue was cut, and DNA was extracted using TIANGEN Genomic DNA Kit according to the manufacturer's instructions. The expression level of H.pylori was detected by qPCR and normalized to GAPDH.The primers(Genewiz) used are listed in Table 1. 
Table 1

Primer sequences

\begin{tabular}{|lll|}
\hline Primer & Forward & Reverse \\
\hline GAPDH & GAGCTGAACGGGAAGCTCAC & AGTGTAGCCCAAGATGCCCT \\
MUC1 & GGCCACCACTCCAGTTTACA & TGCCATTGCTGACTGGAGTT \\
S100A8 & ACAAGGAAATCACCATGCCCT & TTGTGAGATGCCACACCCAC \\
Angiogenin-4 & ACATCAGGGCCATCTGTGGA & GACAGGCCAGCCATCTTCAC \\
Regllly & CCCGTGCCTATGGCTCCTAT & GGCCACTGTTACCACTGCTT \\
H.pylori & TTTGTTAGAGAAGATAATGACGGTATCTAAC & CATAGGATTTCACACCTGACTGACTATC \\
\hline
\end{tabular}

\section{Experimental colitis}

Mice were given $3 \%$ dextran sodium sulphate(DSS,MPbio) solution as drinking water for 7 days to induce acute colitis and then administrated with $1 \times 10^{9} \mathrm{CFU}$ B.fragilis for 3 days. The feces was observed and weight curve was determined by weighing mice daily at the same time throughout the whole experiment. At sacrifice,measure the length of mice colons, $1 \mathrm{~cm}$ was taken from the end of the colons without removing the intestinal contents to ensure the original tissue shape and placed in $4 \%$ paraformaldehyde solution for histopathological analysis.

\section{S rRNA gene sequencing}

The feces samples of all groups were taken and prepared for $16 \mathrm{~S}$ rRNA gene sequencing. The FastDNA ${ }^{\circledR}$ Spin Kit for Stool was used to extract DNA of the feces samples. The detailed operation method was referred to the instruction manual and the main steps are as follows: Feces were pretreated by adding $2 \mathrm{~mL}$ cracking matrix E solution;Centrifuge to remove the pre-cracking buffer; Add MT buffer and sodium phosphate buffer,then homogenize;After full cracking, supernatant is centrifuged; The V4 region of 16S rRNA gene was amplified by using 515F/806R primers (5'-GTGCCAGCMGCCGCGGTAA-3'; $5^{\prime}$-GGACTACHVGGGTWTCTAAT$\left.3^{\prime}\right)$. PCR condition is pre-denaturing at $95^{\circ} \mathrm{C}$ for $2 \mathrm{~min}$, then $95^{\circ} \mathrm{C}$ for $30 \mathrm{~s}$, annealing at $60^{\circ} \mathrm{C}$ for $15 \mathrm{~s}, 72^{\circ} \mathrm{C}$ extending for $30 \mathrm{~s}$, a total of 30 cycles,finally extending at $72^{\circ} \mathrm{C}$ for 5 min.PCR products were detected by $1.5 \%$ agarose gel electrophoresis, and the concentration of DNA was quantified by QIA-Quick PCR Purification Kit.According to the characteristics of the amplified $16 \mathrm{~S}$ region, a small fragment library was constructed and double-end sequencing(Paired_End) was performed at the Illumina Novaseq sequencing platform.

\section{Sequencing data analysis}

Stitching and filtering the Raw Data obtained by the gene sequencing to get Clean Data.Then,OTUs(Operational Taxonomic Units) clustering and species classification were analyzed based on the effective data.According to the results of OTUs, species annotation was made for the representative sequences of each OTU to obtain the corresponding species information and species-based abundance distribution. Meanwhile,the abundance,alpha diversity and beta diversity of OTUs were calculated. Venn plot and petal plot were analyzed to obtain the species richness and evenness in samples,as well as common or 
unique OTUs between different samples or groups, etc.In addition,Principal Component Analysis (PCA) was used for dimensionality reduction analysis to explore the differences of community structure among samples and groups.Finally,statistical analysis such as T-test, Lefse Anosim, MetaStat and MRPP were used to test differences in species composition and community structure of samples.The 16S rRNA sequencing raw data has been uploaded and stored in NCBI Sequence Read Archive(SRA) database.The BioProject accession number was PRJNA699675 and SRA accession number was SRP305360.

\section{SCFAs quantification}

Concentrations of SCFAs in stool samples were detected by gas chromatography.Take $500 \mu \mathrm{L}$ standard or $10 \%$ fecal suspension into $1.5 \mathrm{~mL}$ centrifuge tubes, then add $100 \mu \mathrm{L}$ mixture of crotonic acid and metaphosphoric acid (sample to internal standard ratio 5:1) ;Swirl evenly and put into $-20^{\circ} \mathrm{C}$ overnight; After thawing, centrifuge at 14,000 rpm for 5 min;Take all the supernatant, filter through the water phase filter membrane into a new tube;Add $100 \mu \mathrm{L}$ filtrate to the sample bottle and drain the bubbles from the bottom of the tube, then load the sample and detect $\square$ Standard dilution: dilute the prepared standard solution according to $1 / 2,1 / 5,1 / 10,1 / 20,1 / 100$ and $1 / 500$ of the initial concentration. Then,each standard sample was tested for three times using the same method as described above.The concentrations of SCFAs were calculated by comparing the peak area of the standard and samples, and was expressed as $\mu \mathrm{mol} / \mathrm{g}$ feces.

\section{Tissue lymphocyte isolation}

Mice colon and stomach tissue were opened longitudinally and washed in phosphate buffer solution(PBS);Then put into $14.25 \mathrm{~mL} 1640$ medium containing $10 \mathrm{mM}$ HEPES, 4 mM EDTA, $0.5 \mathrm{mM}$ dithiothreitol(DTT), and $10 \% \mathrm{FBS}$;Shocked at $250 \mathrm{rpm}, 37^{\circ} \mathrm{C}$ for 30 minutes to collect epithelial cells;Filter through $70 \mu \mathrm{m}$ cell strainer and collect the supernatant;Cut up the tissue and add another $14.25 \mathrm{~mL}$ medium solution, the remaining cells were harvested according to the previous method;Tissue lymphocytes were finally extracted by density gradient centrifugation using Percoll(GE) and collected for Flow cytometry analysis.

\section{$\mathrm{CD4}^{+} \mathrm{T}$ cells purification}

The spleen of WT mice was taken and gently ground with a 300-mesh screen in PBS to form suspension,then filtered.Centrifugate to collect cell precipitates.CD4 ${ }^{+} \mathrm{T}$ cells were isolated by MojoSort ${ }^{\mathrm{TM}}$ Mouse CD4 T Cell Isolation Kit(Biolegend). Flow cytometry analysis was performed to detect the proportion of $\mathrm{CD}^{+} \mathrm{T}$ cells, and the subsequent experiments were carried out when the sorting rate reached at least $90 \%$.

\section{siRNA knockdown}

$\mathrm{CD}^{+} \mathrm{T}$ cells were harvested and transfected with siRNA against Smad3(Biomics) in vitro to filter out the valid sequences.After results were confirmed by qPCR and WB,experimental colitis mice were given the modified siRNA intravenously on days 8-10 in vivo.Then inoculated with B.fragilis as described previously.

\section{Flow cytometry analysis}


The proportion and quantity of Tregs were analysed by stained with anti-mouse CD4(GK1.5,Biolegend) and anti-mouse Foxp3 antibody(FJK-16s, eBioscience). For Th1 and Th17 analysis,samples were first stimulated overnight with Cell Activation Cocktail(with Brefeldin A)(Biolegend) in $\mathrm{CO}_{2}$ incubator to induce the intracellular cytokines secreting .After stained with anti-mouse CD4 antibody,cells were fixed and permeabilized by Intracellular Staining Fixation/Permeabilization Buffer (BioLegend), then stained intracellularly with anti-mouse IFN- $\gamma$ (XMG1.2,Biolegend) antibody and anti-mouse IL-17 antibody(9B10,Biolegend).The expression level of colonic IL-10 was detected by using Multifactor kit according to manufacturer's instructions.All experiments were performed on BD Calibur or BD Celesta,and data was analyzed by FlowJo.

\section{RNA extraction and Quantitative Real-time PCR}

Total RNA was extracted from colon or stomach samples with Trizol extraction (Invitrogen,USA).Quantitative Real-time PCR was performed using ABI StepOnePlus Real-Time PCR System.Gene expression level was normalized to GAPDH.The primer sequences(Genewiz) are listed in Table 1.

\section{Western blotting analysis}

Total proteins of colon tissue were extracted by RIPA buffer supplemented with phosphatase inhibitor and protease inhibitor(all from Beyotime), then subjected to western blotting analysis with primary antibodies against p-Smad3 and Smad3(Cell Signaling Technology) in 1:1000.Next,incubated with secondary antibodies(Affinity) in 1:5000.GAPDH(Proteintech) was used as an internal control.Detections of protein expression were performed by Tanon 5200 infrared image system(Tanon Technology).

\section{Tissue ELISA of TGF- $\beta$}

Colons were homogenized in $300 \mu \mathrm{L}$ RIPA Buffer(Beyotime).After incubated at $4^{\circ} \mathrm{C}$ for 30 min to be fully cracked,suspensions were centrifuged at $4^{\circ} \mathrm{C}, 12000 \mathrm{rpm}$ for $15 \mathrm{~min}$. The proteins in supernatant were quantified using BCA Protein Assay Kit (BioRad), then the concentration of TGF- $\beta$ was measured by Mouse TGF- $\beta$ ELISA Kit(MultiSciences) performed as manufacturer's instructions.

\section{Immunofluorescence and Hematoxylin-eosin staining}

Colon and stomach tissue were first fixed in $4 \%$ paraformaldehyde overnight at room temperature or $4^{\circ} \mathrm{C}$.After dehydrated,paraffin-embedded and sliced, samples were stained with hematoxylin and eosin(HE).The representative pictures were recorded by fluorescence inversion microscope to analyze the histopathological characteristics of groups.For immunofluorescence analysis,samples were stained with anti-mouse CD4 and anti-mouse ZO-1 antibody(eBioscience).All antibodies were diluted in 1:100. Cell nuclei were counterstained with DAPI(Sigma).

\section{Statistical analysis}

Statistical analysis was performed with GraphPad Prism 7.0. The differences between groups were analyzed by using ANOVA or $t$ test. $P<0.05$ was regarded as statistically significant.

\section{Results}


In order to explore alteration of intestinal immune response in mice under conditions of intestinal inflammation or treatment.First, we constructed a mouse colitis model using $3 \%$ DSS solution for 7 days.Mice receiving normal water were used as the negative control group,the other group was DSStreated. The weight of the mice was recorded at the same time every day, colon length was measured and HE staining was performed when sacrificed. As shown in Fig. 1, DSS-treated mice showed significant intestinal inflammation,as indicated by weight loss (Fig. 1a) and reduced colon length (Fig. 1b). Further, histopathological analysis of colon tissue revealed that increased intestinal pathology was observed, including inflammatory cells infiltration and absence of crypts (Fig. 1C).

Upon establishment of DSS-induced colitis, the effects of B.fragilis administration were evaluated. Colitic mice were treated with $B$.fragilis by oral gavage for 3 days according to the scheme described in Fig. $2 a$. Fresh feces were collected after the gavages and prepared for 16S rRNA gene sequencing analysis. The alpha diversity analysis of intestinal flora,namely species diversity and abundance of microbial communities within samples,showed that although individual differences were obvious in a group,the diversity and abundance of microbiota in DSS-treated mice were decreased, but increased upon B.fragilis treatment as reflected by the observed species and Chao 1 index (Fig. 2c). The results of beta diversity analysis also revealed the diversity of intestinal flora in the treatment group was significantly improved (Fig. 2d).Dimensionality reduction of sequencing data by Principal Component Analysis (PCA) was used to monitor structural changes in gut microbiota between groups. Differences in microbiota composition of mice with or without $B$.fragilis administration were observed at principal components, while the cluster circle of the B.fragilis-treated mice was overlapped with that of the control group(NC),indicating that its intestinal flora was similar to healthy mice (Fig. 2b). Moreover,the top 10 most abundant species at phylum and genus level were analyzed to compare the relative abundance of species and their proportions.At phylum level, the contraction of Firmicutes and expansion of Verrucomicrobia and Proteobacteria containing pathogenic bacteria were observed in colitic mice.In contrast,gut microbial tends to restore in the mice treated with B.fragilis (Fig. 2e).At the genus level, it was also found that the main microbiota in DSS-colitis changed from Lactobacillus of Firmicutes to Streptococcus, while the Streptococcus was significantly reduced after B.fragilis treatment (Fig. 2f), which are reported to be increased in IBD patients.

Additionally, the analysis of metabolites showed that the production of SCFAs was increased by B.fragilis administration,including acetic acid,propionic acid,butyric acid, isobutyric acid,valeric acid,and isovaleric acid(Fig. 3).Thus, our results demonstrate that B.fragilis therapeutic option during experimental colitis can maintain the microbiota structure and promote SCFAs production.

\section{B.fragilis treatment expands Tregs during protection from colitis}

Due to the strict interaction between intestinal immune system and the host microbiota [10],we next aimed to explore the effects of B.fragilis treatment in intestinal mucosal immune responses. We speculated if the treatment in colitic mice might induce changes in the frequency and function of the colonic infiltrating immune cells.Recent reports have revealed that a critical role of IL-10-producing Tregs in maintenance of 
intestinal immune balance, we thus sought to evaluate how B.fragilis colonization affects the development of Tregs [7].

Next,effects on intestinal immunity in colitic mice treated or not with B.fragilis were evaluated.At sacrifice, B.fragilis-treated mice showed reduced intestinal inflammation, as indicated by decreased weight loss (Fig. 4a) and increased colon length (Fig. 4b). These results were associated to the amelioration of the intestinal inflammation measured by the HE staining of colons and histological score upon B.fragilis (Fig. 4c). More importantly, B.fragilis treatment increased the proportion of Tregs in the colon (Fig. 4d).Meanwhile,the expression of IL-10, a key anti-inflammatory cytokine which contributed to the inflammation resolution, was upregulated(Fig. 4e).

In addition, the functions of intestinal mucosal barrier are closely related to intestinal immunity [11]. The intercellular tight junction is the most important component of gut barrier,and among which the tight junction protein ZO-1 plays a vital role.To evaluate whether $B$.fragilis might exert protective effects on gut barrier, the expression of colonic ZO-1 was tested by immunofluorescence.Consistently, ZO-1 expression level was obviously increased when administrated with B.fragilis in colitic mice, suggesting that the treatment can enhance the intestinal mucosal barrier function(Fig. 4 f).

Of note, gastrointestinal tract is known to be interlinked and gastrointestinal mucosal immunity is closely related. We also found that $B$.fragilis treatment might have the potential to enhance adaptive immunity of stomach and produce immune protection against H.pylori infection in H.pylori-induced gastritis,as demonstrated by increased gastric Th1 and Th17 frequencies, reduced H.pylori colonization and lower levels of antibacterial proteins expression,including MUC1, S100A8, Regllly and Angiogenin - 4 when H.pylori was effectively eliminated (Supplementary Fig. 1a-e).

Altogether, these findings suggest that $B$.fragilis administration enhances regulatory immunity in the gut and effectively alleviates intestinal inflammation.

\section{B.fragilis induces Tregs and reduces colitis through TGF- $\beta /$ Smad3 pathway}

Further, the mechanism of B.fragilis regulating intestinal immunity is explored. Due to different inducing factors, naive $\mathrm{CD}^{+} \mathrm{T}$ cells differentiate into various $\mathrm{T}$ cell subsets, including Th1, Th17 effector cells and Tregs with immunosuppressive effect. There is an increasing evidence that the differentiation of $\mathrm{CD} 4^{+} \mathrm{T}$ cells into Tregs is promoted by TGF- $\beta$ signalling $[8,9]$, and Smad3, a key mediator, plays an important role in the TGF- $\beta$-mediated transcriptional regulation[12].We are therefore to examine how Smad3 responses to B.fragilis treatment affect intestinal inflammatory.

In view of the existing studies on this signaling pathway in vivo mainly used Smad3 $3^{-/-}$mice,which have impaired Treg development [13], we herein conducted Smad3-knocked down mice, and then carried out experimental verification on this basis.First,naive $\mathrm{CD} 4^{+} \mathrm{T}$ cells in spleen were isolated from WT mouse by magnetic beads separation, and we ensured at least $90 \%$ sorting rate before the following experiments (Fig. 5a).After the siRNA sequence that could effectively knock down the expression of Smad3 was obtained by transfection with siRNA against $\mathrm{Smad} 3$ in $\mathrm{CD} 4^{+} \mathrm{T}$ cells, mice were injected intravenously with modified 
siRNA in vivo(Fig. 5b).Control,B.fragilis,siSmad3 + B.fragilis and siSmad3 + Control groups were set up. According to WB results (Fig. 5c),compared with the normal B.fragilis group, $\mathrm{p}$-Smad3 expression level was significantly down-regulated in the siSmad3 + B.fragilis group,and that of the siSmad3 + Control mice was also reduced compared to the Control group.Nonetheless, $B$.fragilis-treated mice exhibited an increase in the p-Smad3 expression. Moreover, a marked elevation in the proportion of colonic Tregs was observed in the B.fragilis group,and it was reduced when Smad3 was knocked down (Fig. 5d). The results of colonic HE staining also showed that B.fragilis treatment in the Smad3 knocked-down mice could not play an effective role in alleviating colitis (Fig. 5e).

These data indicate that Smad3 knocked-down can block the role of B.fragilis in increasing intestinal Tregs, that is to say, Smad3 signaling in B.fragilis administration is indispensable for inducing Tregs and provides a molecular mechanism of protection from colitis.

\section{B.fragilis activates TGF- $\beta$ through increased ROS}

Our previous date indicated that the frequency of Tregs was significantly increased upon B.fragilis treatment.Interestingly, Treg differentiation was induced by TGF- $\beta$. Reactive oxygen species(ROS) has been reported to be one of the factors that activate TGF- $\beta[14,15]$. Thus, we suspected if $B$. fragilis in mouse models of intestinal inflammation might have an effect on ROS production to activate TGF- $\beta$.

To test our hypothesis, we constructed the mouse colitis models and given B.fragilis treatment as previously described.As expected,higher amounts of ROS (Fig. 6a) as well as increased expression of TGF- $\beta$ (Fig. 6b) were observed in the colons of mice with B.fragilis-treated as compared to DSS-treated mice in our further study.In vitro,blockade of ROS was shown to prevent Th17 generation when naive CD4 ${ }^{+} \mathrm{T}$ cells were cultured with latent TGF- $\beta$ and IL-6 [16],suggesting that ROS plays an important role in TGF- $\beta$ activation.These results highlight that the increased ROS by $B$.fragilis may greatly contribute to activating TGF- $\beta$,thus induces the expansion of Tregs to enhance intestinal immune tolerance.

Taken together, our data show that the restoration of gut microbiota by B.fragilis exerts a profound impact on the intestinal immunity. The microbiota alteration is pivotal to the beneficial anti-inflammatory effects,supported by the increase of Treg frequency,colonic IL-10 and ZO-1 expression.Most importantly, B.fragilis-induced ROS production may be responsible for the observed therapeutic effects by activating TGF- $\beta$ to induce Smad3-dependent Treg differentiation,thus maintains intestinal homeostasis.

\section{Discussion}

Faecal microbiota transplantation(FMT) is becoming a research hotspot as an effective therapeutic approach to control intestinal inflammation in recent years [1]. FMT has been clinically used in treating intestinal diseases that associated with microbiota due to fewer side effects[17,18].However,data on its mechanism of playing a role in immune regulation are still scarce.Studies have shown that,the gut microbiota in healthy mice was composed of a similar core microbial ecology, including Firmicutes and Bacteroidetes. Our previous work also demonstrated that GEMs administration could change the intestinal flora diversity and increase the relative abundance of Bacteroidetes[3].Current theories suggest that 
B.fragilis,a member of Bacteroidetes,can induce $\mathrm{CD}^{+}{ }^{+} \mathrm{Foxp}^{+}$regulatory $\mathrm{T}$ cells to reduce intestinal inflammation[19].Although it is known that B.fragilis treatment has protective effects on the immune system,the pathways by which it regulates gastrointestinal immunity is still unclear .

In our study, $16 \mathrm{~S}$ rRNA gene sequencing analysis of the gut microbiota in colitic and B.fragilis-treated mice showed that $B$.fragilis administration for 3 days are sufficient to implement the transition of intestinal flora to homeostasis $[20,21]$.The relative abundance of the core bacteria,Firmicutes and Bacteroidetes,were increased upon B.fragilis during colitis.Consistently, the reduction of Verrucomicrobia and Proteobacteria were observed as expected.Further analysis at the genus level also found that the exogenous administration of B.fragilis could effectively reverse the shift from normobiosis to dysbiosis in mice with colitis.In addition,the increase of SCFAs production was confirmed in B.fragilis treatment.

A healthy gut environment, shaped by a healthy microbiota, is necessary for ensuring immune balance. Many reports indicate that $\mathrm{CD} 4^{+} \mathrm{T}$ cells play a pivotal role in the initiation and development of intestinal inflammation as its excessive production. Of note,mice with inflammatory bowel disease show increased amounts of intestinal Th1, Th17 and Th1/17 cells that promote the inflammatory process [22,23].In addition to the subsets as described,the expression of Tregs derived from CD $4^{+} \mathrm{T}$ cells and IL-10,a anti-inflammatory cytokine,suggest that the alteration in the gut microbiota during colitis or upon B.fragilis administration is greatly related to the changes in the mucosal immune responses.Moreover, the option also has an effect on intestinal barrier function as indicated by a higher level of ZO-1 in the colon.Interestingly,we also found that variations in the intestinal microbial ecology are capable to provide protection from gastritis induced by H.pylori.The analysis of Th1 and Th17 cells,H.pylori colonization and antibacterial proteins in the stomach were all shown in detail.

Further,our experiments indicated that Treg expansion might be critically involved in the tolerogenic mechanisms triggered by therapeutic B.fragilis during experimental colitis. The production of gut microbiotaderived SCFAs was significantly increased. Interestingly,high free fatty acid stimulation results in the expression of highly reactive ROS [24].Moreover,ROS is known to activate TGF- $\beta$,even if only a few TGF- $\beta$ activation pathways are known. We illustrated that $B$.fragilis-induced SCFAs might activate TGF- $\beta$ through increased ROS and then induced the Treg expansion,as higher levels of colonic ROS and TGF- $\beta$ were shown after the treatment.More importantly, expansion of Tregs in B.fragilis-treated mice with smad3 knocked down was prevented,confirming a prominent role of TGF- $\beta /$ Smad3 signaling pathway in immune modulation induced by $B$.fragilis.Further study will explore if other immune pathways or cell communities may contribute to the tolerogenic mechanisms in the gut.

In conclusion,we demonstrate that the restoration of gut microbiota by B.fragilis treatment during colitis shows various effects on both regulatory and adaptive intestinal immune.The differentiation of Tregs,induced by activated TGF- $\beta$ via TGF- $\beta$ /Smad3 signaling, contributes to the resolution of inflammation. Our findings on overall provide a mechanism explanation of $B$.fragilis treatment and suggest its potential to be a probiotic product in the future.

\section{Declarations}




\section{AUTHOR CONTRIBUTIONS}

All authors contributed to the study conception and design. Material preparation, data collection and analysis were mainly performed by M.H.F. The first draft of the manuscript was written by M.H.F. and all authors commented on previous versions of the manuscript. All authors read and approved the final manuscript.

DATA AVAILABILITY

\section{All data generated or analysed during this study are included in the article and its supplementary information files.}

\section{DECLARATIONS}

Ethics approval and consent to participate. All animal experiments were performed in accordance with guidelines of the Animal Ethical and Experimental Committee of China Pharmaceutical University.

Consent for publication. Not Applicable.

Funding. This work was supported by National Key Research and Development Project (No. 20 17YFD0400303),National Natural Science Foundation of China (No.

81971562),Key R \& D Plan Project of Ningxia Autonomous Region (No.2020BFG02012), The Priority Academic Program Development (PAPD) of Jiangsu Higher Education Institutions.

Acknowledgements. Thanks for the National Key Research and Development Project and National Natural Science Foundation of China.

Competing interests. The authors declare no competing interests.

\section{References}

1.Burrello C, Garavaglia F, Cribiù FM, Ercoli G, Lopez G, Troisi J, et al. (2018) Therapeutic faecal microbiota transplantation controls intestinal inflammation through IL10 secretion by immune cells. Nat Commun 9(1):5184. https://doi.org/10.1038/s41467-018-07359-8

2.Liu W, Tan Z, Xue J, Luo W, Song H, Lv X, et al. (2016) Therapeutic efficacy of oral immunization with a non-genetically modified Lactococcus lactis-based vaccine CUE-GEM induces local immunity against Helicobacter pylori infection. Appl Microbiol Biotechnol 100(14): 6219-6229.

https://doi.org/10.1007/s00253-016-7333-y

3.Zeng Z , Liu W , Luo S ,Hu C , Xu N , Huang A, et al. (2019) Shape of gastrointestinal immunity with nongenetically modified Lactococcus lactis particles requires commensal bacteria and myeloid cells-derived TGF-B1. App/ Microbiol Biotechnol 103(9):3847-3861. https://doi.org/ 10.1007/s00253-019-09716-z 
4.Wexler HM. (2007) Bacteroides: the good, the bad, and the nitty-gritty. Clin Microbiol Rev 20(4): 593-621. https://doi.org/10.1128/CMR.00008-07

5.Mazmanian SK, Round JL, Kasper DL. (2008) A microbial symbiosis factor prevents intestinal inflammatory disease. Nature 453(7195):620-5. https://doi.org/10.1038/nature07008

6.Lee YP, Huang WC, Lin TJ, Chiu CC, Wang YC, Chen YH, et al. (2020) Toll-like receptor 4 prevents AOM/DSS-induced colitis-associated colorectal cancer in Bacteroides fragilis gnotobiotic mice. Hum Exp Toxicol 14:960327120954249. https://doi.org/10.1177/096032712095 4249

7.Rubtsov YP, Rasmussen JP, Chi Emil Y, Fontenot J, Castelli L, Ye X, et al. (2008) Regulatory T cell-derived interleukin-10 limits inflammation at environmental interfaces. Immunity 28(4):546- 58. https://doi.org/10.1016/j.immuni.2008.02.017

8.Yang L,Pang Y.,Moses HL. (2010) TGF-beta and immune cells: an important regulatory axis in the tumor microenvironment and progression. Trends Immuno/ 31(6):220-227. https://doi.org/ 10.101 6/j.it.2010.04.002

9.Flavell RA, Sanjabi S, Wrzesinski SH, Licona-Limón P. (2010) The polarization of immune cells in the tumour environment by TGFbeta. Nat Rev Immuno/ 10(8):554-67. https://doi.org/10.1038/ nri2808

10.Macpherson AJ, Harris NL. (2004) Interactions between commensal intestinal bacteria and the immune system. Nat Rev Immunol 4(6):478-85. https://doi.org/10.1038/nri1373

11.Sánchez de Medina F, Romero-Calvo I, Mascaraque C, Martínez-Augustin O. (2014) Intestinal inflammation and mucosal barrier function. Inflamm Bowel Dis 20(12):2394-404. https://doi.org/ 10.1097/MIB.0000000000000204

12.Datto MB, Frederick JP, Pan L,Borton AJ,Zhuang Y, Wang XF. (1999) Targeted disruption of Smad3 reveals an essential role in transforming growth factor beta-mediated signal transduction. $\mathrm{Mol} \mathrm{Cell} \mathrm{Biol}$ 19(4):2495-504. https://doi.org/10.1128/mcb.19.4.2495

13.Gu AD, Wang Y, Lin L, Zhang SS, Wan YY. (2012) Requirements of transcription factor Smad- dependent and -independent TGF-b signaling to control discrete T-cell functions. Proc Natl Acad Sci U S A 109(3):90510. https://doi.org/10.1073/pnas.1108352109

14.Cruz-Bermúdez A, Laza-Briviesca R, Vicente-Blanco RJ, García-Grande A, Coronado MJ, Laine-Menéndez $S$, et al. (2019) Cancer-associated fibroblasts modify lung cancer metabolism involving ROS and TGF-beta signaling. Free Radic Biol Med 130:163-173. https://doi.org/10.101 6/j.freeradbiomed.2018.10.450

15.Khalil N. (1999) TGF-beta: from latent to active. Microbes Infect 1(15):1255-63. doi:10.1016/ s12864579(99)00259-2

16.Zhang D, Jin W, Wu R, Li J, Park SA, Tu E, et al. (2019) High Glucose Intake Exacerbates Autoimmunity through Reactive-Oxygen-Species-Mediated TGF-b Cytokine Activation. Immunity 51(4):671-681.e5.

Page 12/19 
doi:10.1016/j.immuni.2019.08.001

17.Cammarota G, laniro G, Tilg H, Rajilić-Stojanović M, Kump P, Satokari R, et al. (2017) European consensus conference on faecal microbiota transplantation in clinical practice. Gut 66(4):569-580. https://doi.org/10.1136/gutjnl-2016-313017

18.van Nood E, Vrieze A, Nieuwdorp M, Fuentes S, Zoetendal EG, de Vos WM, et al. (2013) Duodenal infusion of donor feces for recurrent Clostridium difficile. N Engl J Med368(5):407-415. https://doi.org/10.1 056/NEJMoa1205037

19.Round JL, Mazmanian SK. (2010) Inducible Foxp3+ regulatory T-cell development by a commensal bacterium of the intestinal microbiota. Proc Natl Acad Sci U S A 107(27):12204-9.

https://doi.org/10.1073/pnas.0909122107

20.Paramsothy S, Kamm MA, Kaakoush NO, Walsh AJ, van den Bogaerde J, Samuel D, et al. (2017) Multidonor intensive faecal microbiota transplantation for active ulcerative colitis: a randomised placebocontrolled trial. Lancet 389(10075):1218-1228. https://doi.org/10.1016/S014 0-6736(17)30182-4

21.Rossen NG, Fuentes S, van der Spek MJ, Tijssen JG, Hartman JH, Duflou A, et al. (2015) Findings from a randomized controlled trial of fecal transplantation for patients with ulcerative colitis. Gastroenterology 149(1):110-118.e4. https://doi.org/10.1053/j.gastro.2015.03.045

22. Nistala K, Adams S, Cambrook H, Ursu S, Olivito B, de Jager W, et al. (2010) Th17 plasticity in human autoimmune arthritis is driven by the inflammatory environment. Proc Natl Acad Sci U S A 107(33):1475114756. https://doi.org/10.10 73/pnas.1003852107

23.Fujino S, Andoh A, Bamba S, Ogawa A, Hata K, Araki Y, et al. (2003) Increased expression of interleukin 17 in inflammatory bowel disease. Gut 52(1):65-70.https://doi.org/10.1136/gut.52.1.65

24.Qi Y, Du X, Yao X, Zhao Y. (2019) Vildagliptin inhibits high free fatty acid (FFA)-induced NLRP3 inflammasome activation in endothelial cells. Artif Cells Nanomed Biotechnol 47(1):1067-1074. https://doi.org/10.1080/21691401.2019.1578783

\section{Figures}


a

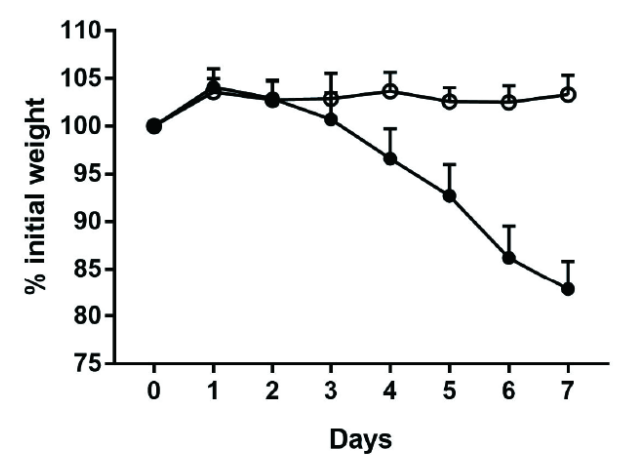

C

NC

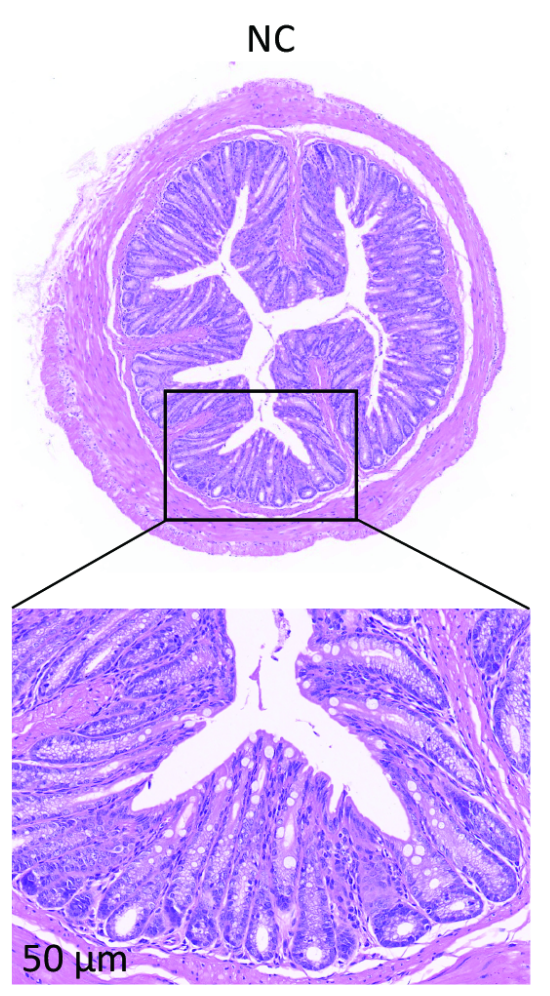

b

$-N C$
$\rightarrow$ DSS
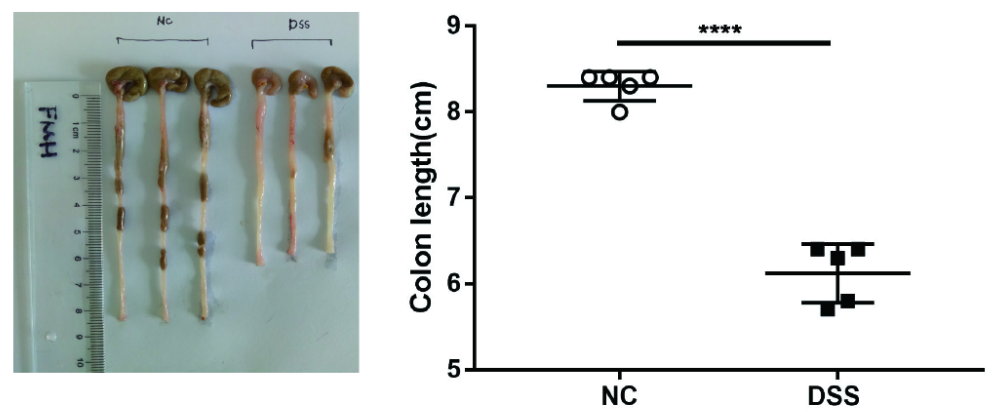

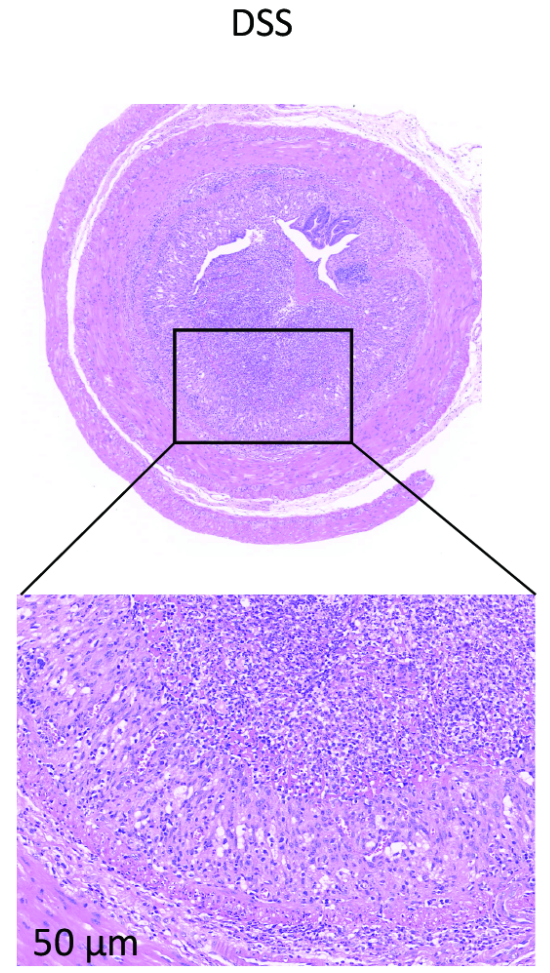

\section{Figure 1}

Establishment of mouse colitis model. a,b Weight loss (a) and colon length (b) of untreated (NC) and colitic

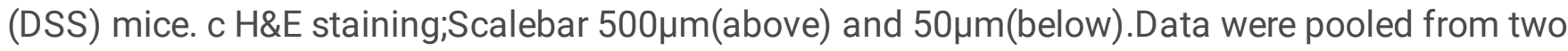
independent experiments ( $n=5$ per group). ${ }^{\star} P<0.05,{ }^{\star *} P<0.01,{ }^{* \star *} P<0.001$ were regarded as statistically significant through ANOVA or t test. Data were represented as mean \pm SD. 
b

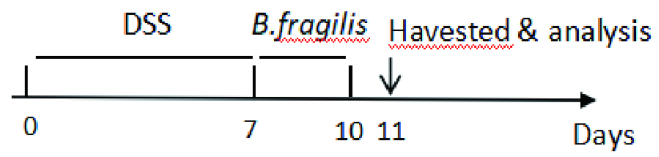

C
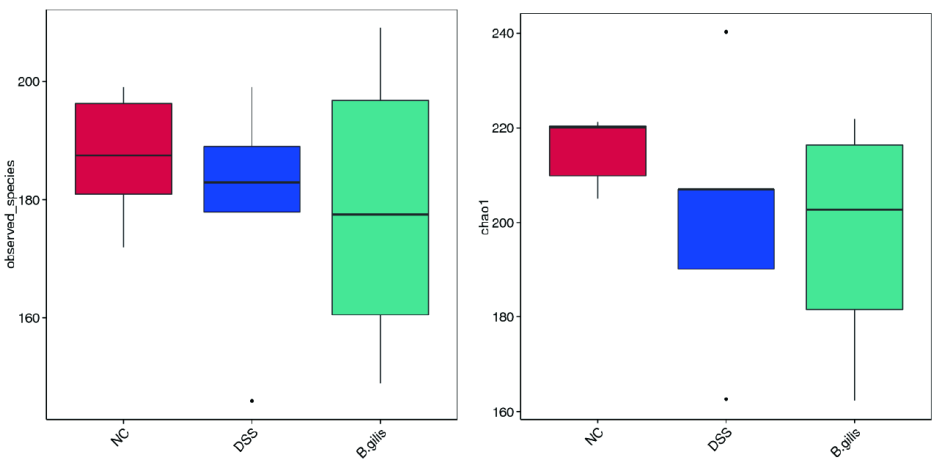

d
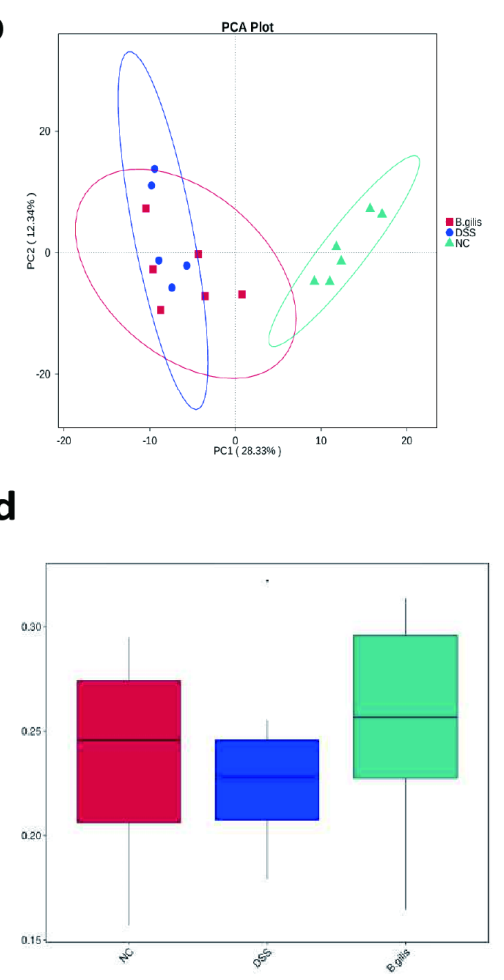

e

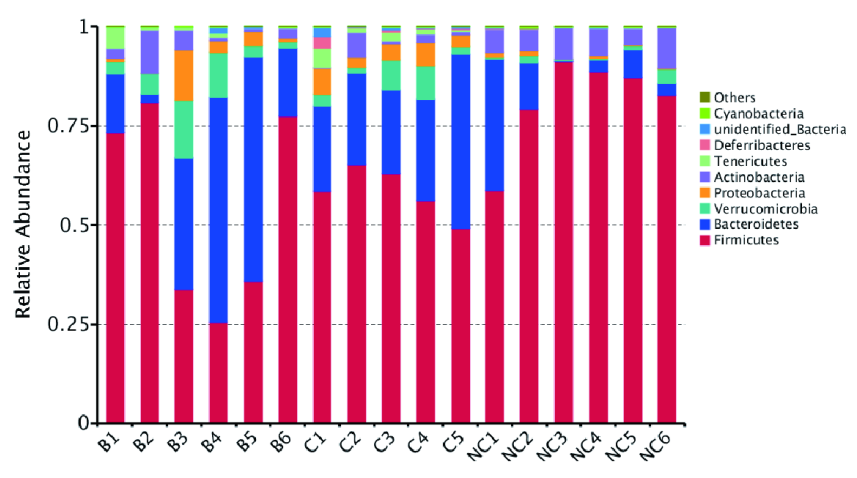

f

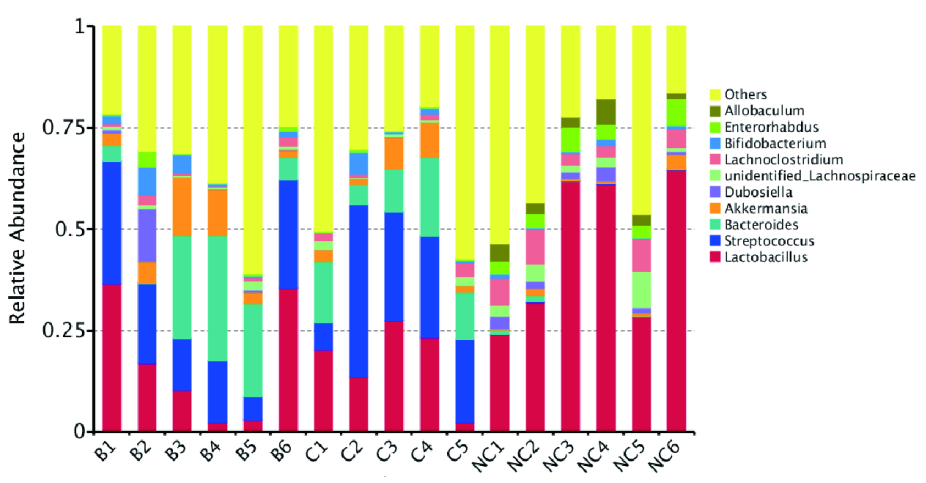

Figure 2

B.fragilis treatment alters intestinal flora. a Schematic representation of B.fragilis treatment during DSS experimental colitis. b Microbiome clustering based on Principal Coordinate Analysis (PCA) UniFrac metrics of fecal gut microbiota derived from DSS, DSS+B.fragilis and NC mice. c Observed species index depicted the microbial diversity and Chao 1 index depicted microbial abundance; B.gilis (DSS +B.fragilis). d Beta diversity analysis of intestinal flora. e,f Relative abundance of the top 10 most abundant OTUs at the phylum(e) and genus(f) level in NC, DSS (C1-5) and DSS+B.fragilis(B1-6) treated mice. Each bar represents 
an individual mouse(NC $n=6$, DSS $n=5$, DSS+B.fragilis $n=6) .{ }^{*} P<0.05,{ }^{*} \times<<0.01,{ }^{*} * x<0.001$ were regarded as statistically significant through ANOVA or t test. Data were represented as mean \pm SD. In Box and whiskers plots, centre line represents median.

a

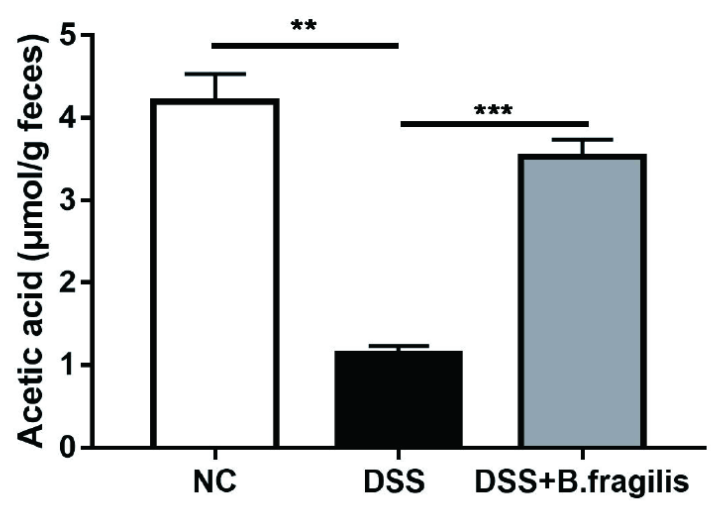

C

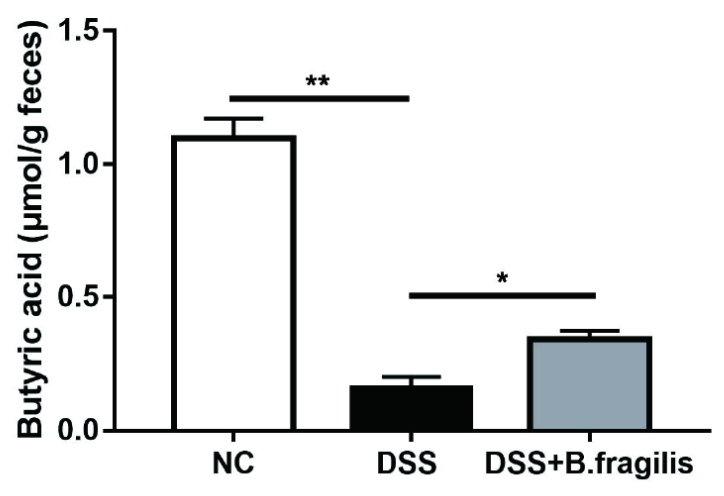

b

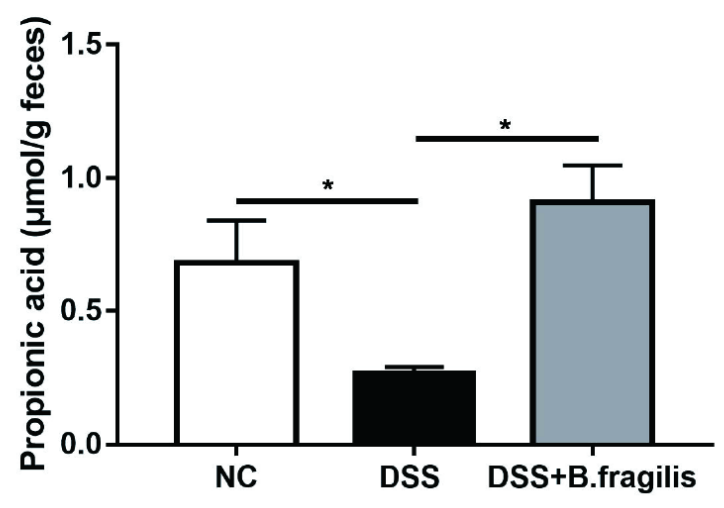

d

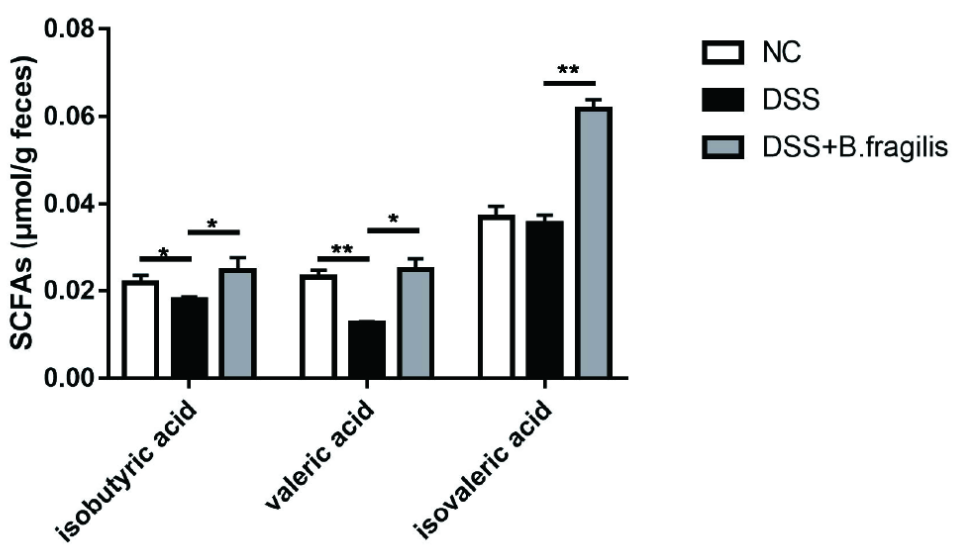

Figure 3

B.fragilis treatment alters SCFAs production. a-d Levels of acetic acid, propionic acid, butyric acid, and other SCFAs (isobutyric acid, valeric acid, isovaleric acid). ${ }^{*} P<0.05$, ${ }^{\star *} P<0.01,{ }^{\star} * \star P<0.001$ were regarded as statistically significant. Data were represented as mean \pm SD. 
a

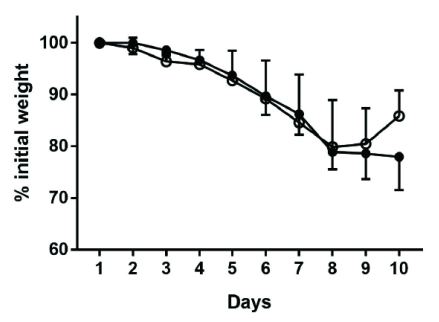

c
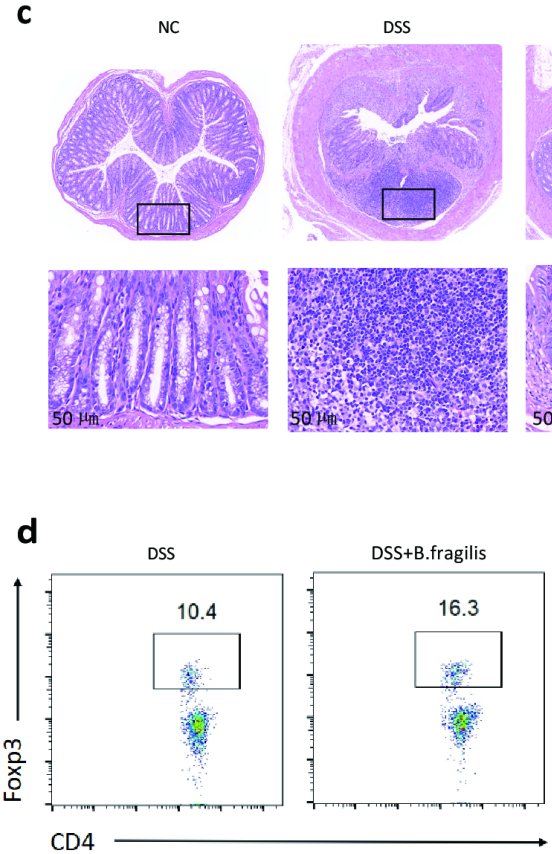

$\rightarrow$ DSS
$\rightarrow$ DSS+B.fragilis

b
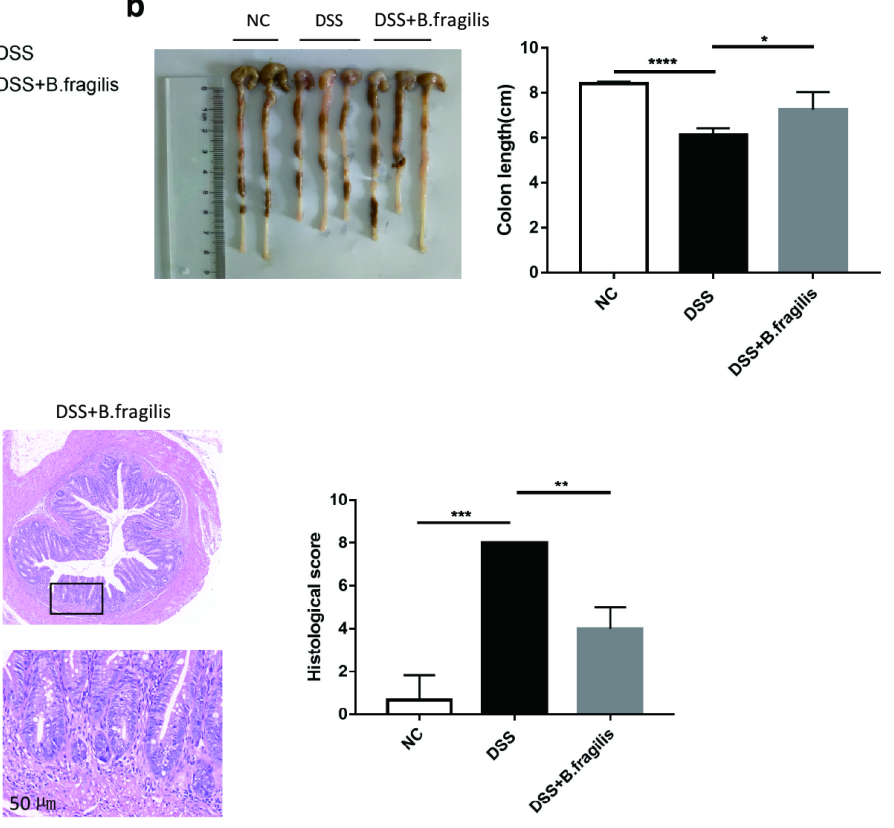

e
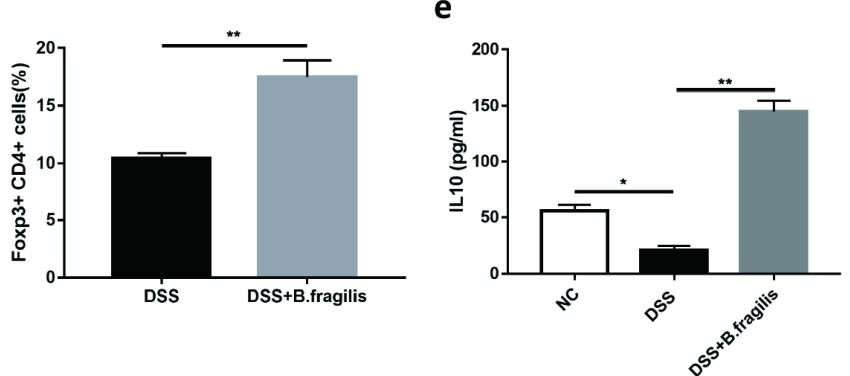

f
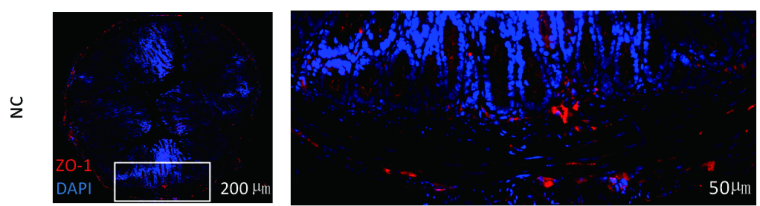

ณิ
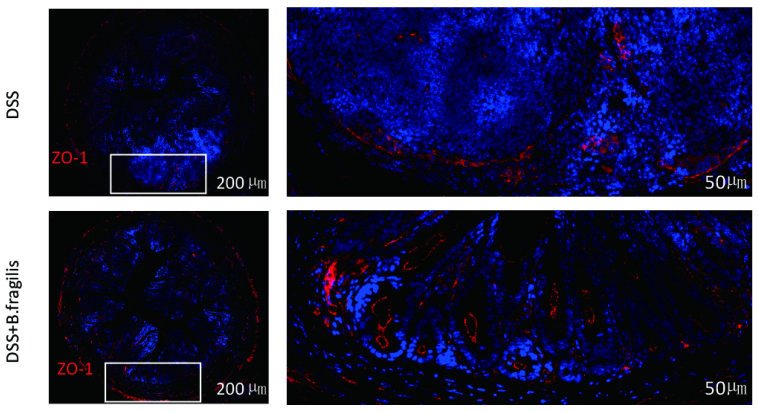

Figure 4

B.fragilis treatment expands Tregs during protection from experimental colitis. a,b Weight loss (a) and colon length (b) of DSS-treated and DSS+B.fragilis-treated mice. c H\&E staining (scale bar 500 $\mu \mathrm{m}$ and $50 \mu \mathrm{m}$ ) and cumulative histological score of colon specimens; Mucosal injury and inflammatory infiltration were included in detailed histological evaluation of mice. $d$ Representative plots, frequencies of colonic CD4+ Foxp3+ Tregs in DSS and DSS+B.fragilis treated mice by flow cytometry. Bar graph shows data from three experiments. e Flow cytometry analysis of colonic IL-10 cytokine level. $\mathrm{f}$ Immunofluorescence detects the 
ZO-1 protein in colon. Representative images stained with the antibodies recognizing ZO-1(red) are shown. Nuclei were counterstained with DAPI (blue). Scale bar, $200 \mu \mathrm{m}$ (left panel) and $50 \mu \mathrm{m}$ (right panel). In (a-f) $n=6$ per group. Data represent mean $\pm S D$. ${ }^{*} P<0.05,{ }^{*} P<0.01,{ }^{*} * P<0.001$ were regarded as statistically significant.

a

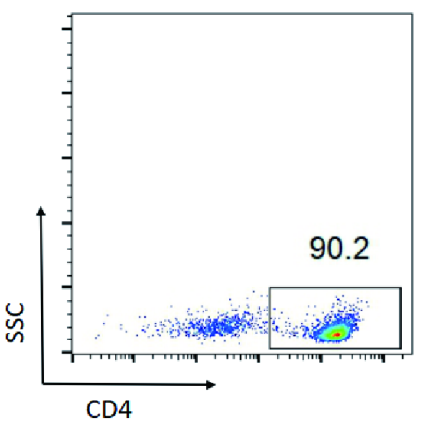

b

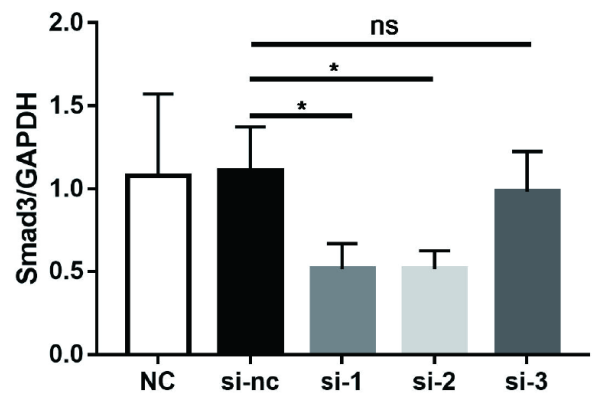

c

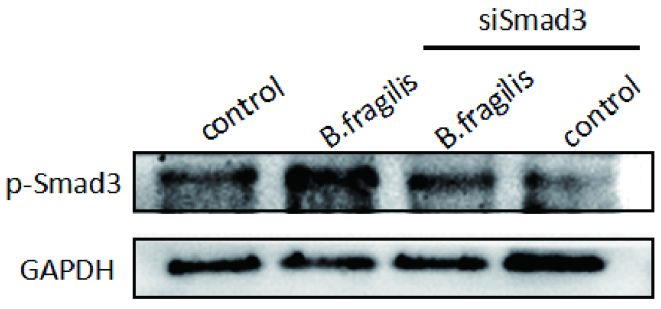

d
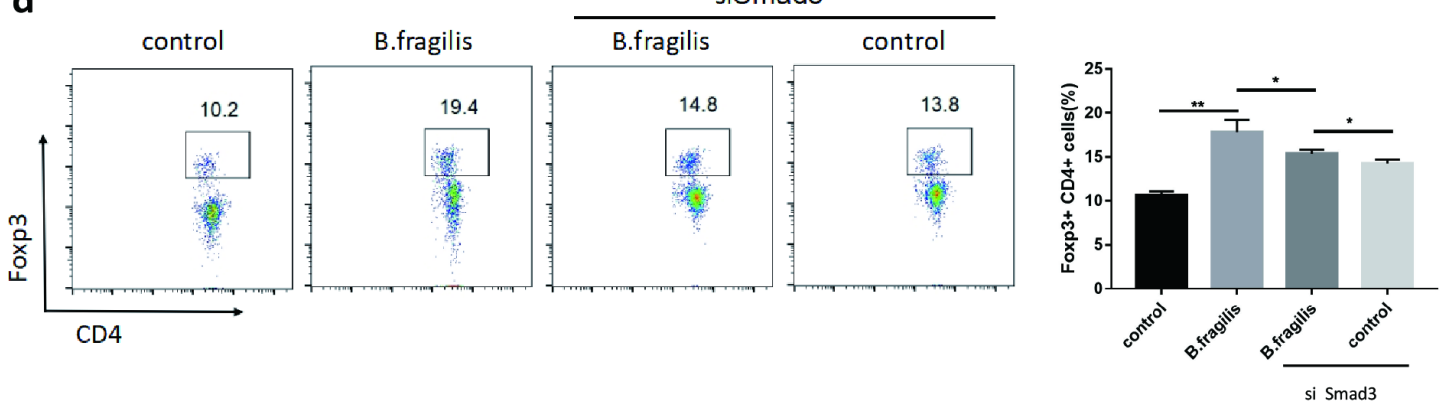

e

siSmad3
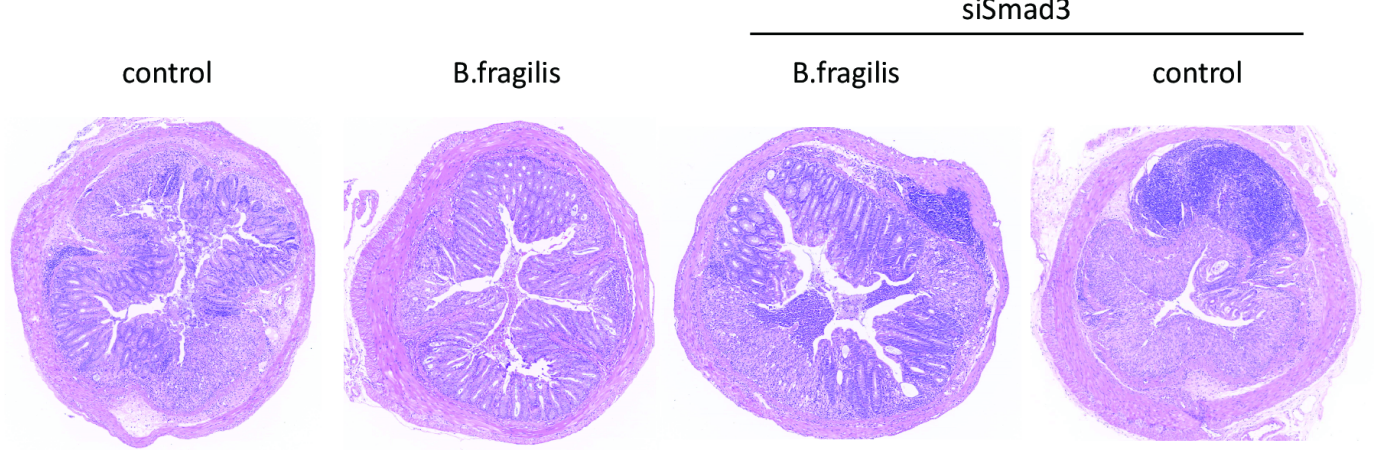

Figure 5

B.fragilis protection from intestinal inflammation requires Smad3.a Naive CD4+ T cells isolated from spleen of WT mice.b Effects of siRNA on Smad3 mRNA expression in spleen-derived naive CD4+ T cells. c Western 
blotting analysis shows the expression of p-Smad3 in the colon isolated from DSS, DSS+B.fragilis,and DSS+B.fragilis or DSS with Smad3 knocked-down mice ( $n=5$ per group).d Flow cytometry analysis shows the effects of knockdown of Smad3 in the production of Tregs in different groups.e Representative H\&E staining of colons. ${ }^{\star} P<0.05,{ }^{*} P<0.01,{ }^{\star} * \star P<0.001$ were regarded as statistically significant.Data were shown as mean \pm SD.

\section{a}
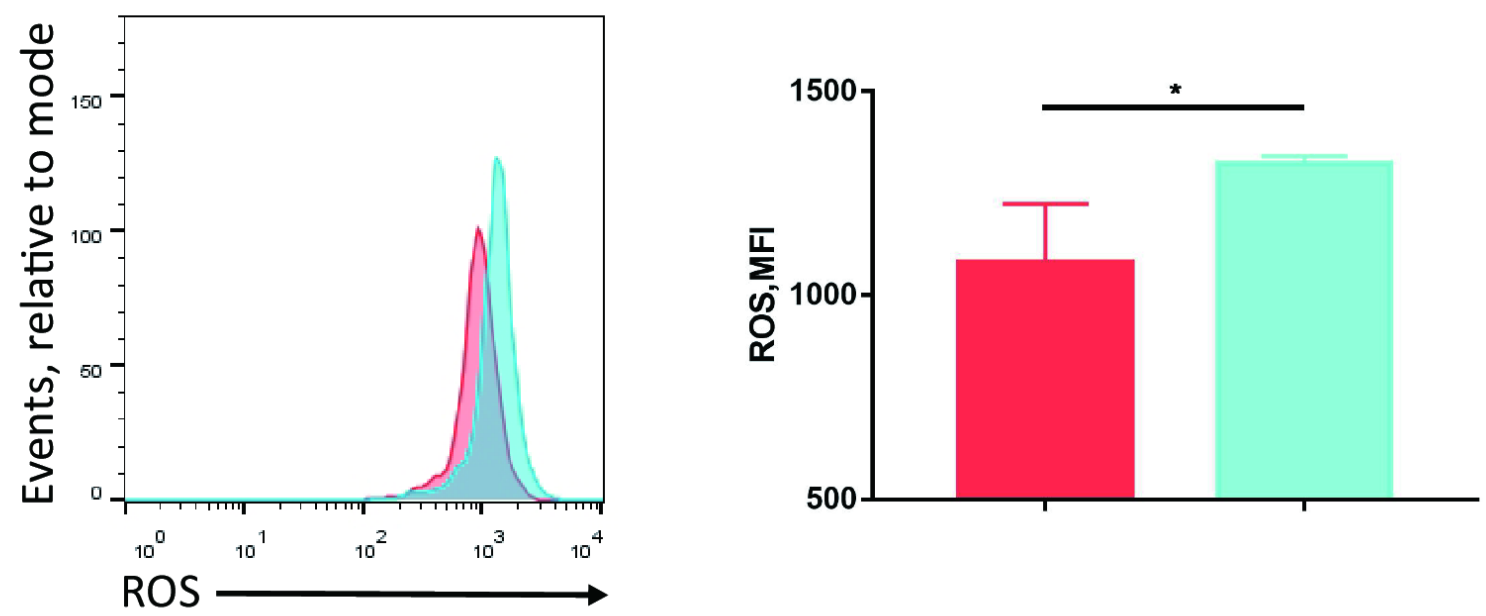

DSS

DSS+B.fragilis

\section{b}

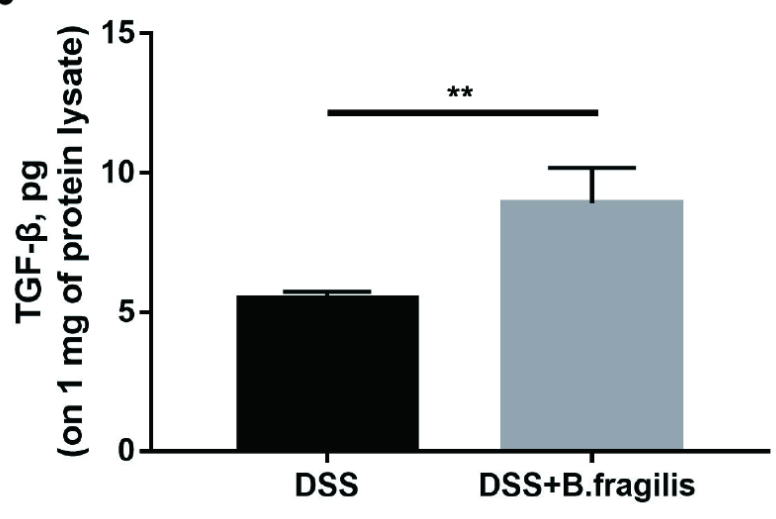

Figure 6

ROS activates TGF- $\beta$. a Representative histograms of ROS expression and cumulative Mean fluorescence intensity (MFI) on colonic CD4+T cells in DSS (red boxes) and DSS+B.fragilis (green boxes) treated mice. $b$ TGF- $\beta$ colonic expression. In (a,b) DSS, DSS+B.fragilis $n=5 ;{ }^{*} P<0.05$, ${ }^{* *} P<0.01,{ }^{*} * \mathrm{P}<0.001$ were regarded as statistically significant through ANOVA or $t$ test. Data were represented as mean $\pm S D$.

\section{Supplementary Files}

This is a list of supplementary files associated with this preprint. Click to download.

- Supplementarymaterial.docx 